Check for updates

Cite this: RSC Adv., 2019, 9, 34607

\title{
Impact of deoxynivalenol and kaempferol on expression of tight junction proteins at different stages of Caco-2 cell proliferation and differentiation $\dagger$
}

\author{
Xiaojie Wang, Li Li and Genyi Zhang (DD*
}

\begin{abstract}
The barrier function of intestinal tract is essential to gut health, and the expression of tight junction (TJ) proteins in human epithelial colorectal adenocarcinoma (Caco-2) cells, mimicking the intestinal stem cell proliferation and differentiation, was investigated after treatment by the mycotoxin of deoxynivalenol (DON) and phenolic compound of kaempferol (KAM). The results showed that DON (5 $\mu$ M) significantly reduced the expression of claudin-4 while kaempferol $(100 \mu \mathrm{M})$ increased the expression of claudin-3 during Caco-2 cell proliferation. After being cultured for 11 days, the DON treatment, unexpectedly, augmented the expression of claudin-4 with an increased TEER. For differentiated Caco-2 cells after a 21 day culture, both the TEER and claudin-4 levels were significantly reduced by DON while KAM pretreatment alleviated the damage caused by DON accompanying an increase of TEER, claudin-3, and ZO-1. Thus, kaempferol and DON differentially affected the expression of tight junction proteins at different stages of Caco-2 cell proliferation and differentiation, and an increase of the integrity of TJ after KAM pretreatment indicates KAM has the potential to protect the integrity of the barrier function of the intestinal epithelium for improved health.
\end{abstract}

Received 9th August 2019
Accepted 18th October 2019

DOI: 10.1039/c9ra06222j

rsc.li/rsc-advances their distribution contribute to the $\mathrm{TJ}$ barrier function. However, literature study using porcine intestinal epithelial cell line showed that mycotoxin of DON decreased the expression of claudin-4 with impaired intestinal barrier function. ${ }^{13}$ Similar result was also observed in differentiated Caco-2 cell monolayer with increased membrane permeability after exposure to DON. ${ }^{5}$ Accordingly, strategies by employment of various bioactive substances to regulate tight junction proteins have become a hot research area. ${ }^{14-18}$ The repair effect of galactooligosaccharide on DON-induced damage in Caco-2 monolayer and mice intestine showed that GOSs stabilized the expression and cellular distribution of claudin-3 in the monolayer Caco-2 cells. ${ }^{19}$ The protective effects of quercetin, naringenin, berberine and curcumin on the integrity of tight junction have all been reported..$^{20-23}$ Gut microbiota fermented product of butyrate also enhances the intestinal barrier function through facilitating the assembly of TJ proteins. ${ }^{24}$ Thus, bioactive compounds might be an effective strategy to combat the toxicity of DON for improved barrier functions of intestinal epithelium to protect the body against luminal bacteria and toxic materials. Kaempferol is a polyphenol antioxidant with a rich content in fruits and vegetables, and its beneficial effects in reducing the risk of chronic diseases have been extensively studied..$^{25}$

Kaempferol can not only augment the body's antioxidant defense system, and modulate key elements of signaling
State Key Laboratory of Food Science and Technology, School of Food Science and Technology, Jiangnan University, Wuxi, Jiangsu 214122, China. E-mail: gzhang@ jiangnan.edu.cn; Tel: +86-510-85328726

$\dagger$ Electronic supplementary information (ESI) available. See DOI: 10.1039/c9ra06222j 
pathways related to cellular apoptosis and inflammation, it also exerts protective effect against toxic materials. ${ }^{26-28}$ As a preserving agent, kaempferol could activate the MAPK pathway and to increase the antioxidant capacity such as the expression of antioxidant enzyme of HO-1. Concerning the protective effect on cellular tight junction, kaempferol could substantially increase the transepithelial electrical resistance (TEER, tight junction marker) in a Caco-2 cell line, promote the protein expression of ZO-1 and -2, occludin, and claudin-1, -3, and -4 , and the actin cytoskeletal association of the tight junction proteins in cholesterol-rich lipid microdomain. ${ }^{29}$ However, to our knowledge, there is no literature reports on the protective effect of kaempferol on the DON-induced TJ damage. Additionally, literature study showed that the TJ complexes of Caco-2 cells at different stages of differentiation had different sensitivity to the bioactive compounds. ${ }^{16}$ Thus, the impact of kaempferol on DON-induced TJ changes was studied at different stages of proliferation and differentiation of Caco-2 cells so that a deeper understanding of the physiological function of kaempferol in protecting the intestinal barrier function will be achieved.

\section{Materials and methods}

\subsection{Chemicals and reagents}

Deoxynivalenol (DON, $\mathrm{C}_{15} \mathrm{H}_{20} \mathrm{O}_{6}$, $\geq 98 \%$ purity) was purchased from Sigma Aldrich (St Louis, MO, USA). Kaempferol $\left(\mathrm{C}_{15} \mathrm{H}_{10} \mathrm{O}_{6}\right.$, $\geq 98 \%$ purity) was purchased from Aladdin Biochemical Technology Co., Ltd (Shanghai, China). Antibodies of $\beta$-actin, claudin-3, claudin-4, occludin and ZO-1 were obtained from Sigma Aldrich (St Louis, MO, USA). Secondary antibodies were obtained from Abcam Co., Ltd (Shanghai, China). Alkaline phosphatase (AKP) activity measurement kit was purchased from Nanjing Jiancheng Bioengineering Institute (Nanjing, China). The NuPAGE® MOPS SDS Buffer Kit, NuPAGE® Bis-Tis Mini Gels and iBlot ${ }^{\circledR} 2$ Transfer Stacks were purchased from Thermo Fisher Scientific (Waltham, MA, USA).

\subsection{Culture of Caco-2 cells}

Human epithelial colorectal adenocarcinoma cells (Caco-2 line, passages 22) obtained from Chinese Academy of Sciences Cell Bank (Shanghai, China) were cultured in $25 \mathrm{~cm}^{2}$ Corning culture flasks (Corning Incorporated, Corning, New York, USA) in Minimum Essential Medium (MEM) (Life Technologies, Inc., Invitrogen, Shanghai, China), and supplemented with $20 \%$ fetal bovine serum (FBS), 1\% penicillin-streptomycin $10000 \mathrm{U}$ $\mathrm{mL}^{-1}$ ), $1 \%$ Glutamax, $1 \%$ sodium pyruvate, and 1\% nonessential amino acids (Life Technologies). Cells were kept at $37^{\circ} \mathrm{C}$ in a humid atmosphere of $5 \% \mathrm{CO}_{2}-95 \%$ air. The trypsinization at cell density of $80 \%$ confluence was performed with $0.25 \%$ trypsin-EDTA (Life Technologies) for about 4 minutes, and a new passage of Caco- 2 cell will be cultured in flasks. The cells between 25-35 passages were used for the experiments.

\subsection{Establishment of Caco-2 cell monolayer model}

Add $600 \mu \mathrm{L}$ and $50 \mu \mathrm{L}$ of culture medium to a 24 well plate and collagen-coated polytetrafluoroethylene (PTFE) insert $\left(0.33 \mathrm{~cm}^{2}\right.$ membrane area with $0.4 \mu \mathrm{m}$ pores, Corning Incorporated, Corning, New York, USA), respectively. Then place them in a $3{ }^{\circ} \mathrm{C}$ incubator for at least $1 \mathrm{~h}$, and digest the fused cells to achieve more than $90 \%$ of single cells. The transwell plate was taken out from the incubator, and $50 \mu \mathrm{L}$ of the cell suspension was added to the inside of the insert to reach a cell density of 1.3 $\times 10^{5}$ cells per $\mathrm{cm}^{2}$. The first medium change will be finished within 16 hours. Medium was then refreshed every other day within the first week, and every day after a week. After 21 day of culture, a confluent monolayer was achieved with a mean transepithelial electrical resistance (TEER) about $260 \pm 65 \Omega$ $\mathrm{cm}^{2}$ measured by a EVOM2 epithelial cell transmembrane resistance meter (WPI, Shanghai, China). The experiment can be carried out within one week thereafter, and the medium needs to be changed 12-24 hours before the experiment.

\subsection{TEER measurement}

The AP side and the BL side of the cultured Caco- 2 cells were washed with a Hank's solution $(\mathrm{pH} 7.4)$ to remove cell monolayer surface attachments. Then $0.1 \mathrm{~mL}$ and $0.6 \mathrm{~mL}$ Hank's solution were added to inside and outside of the insert, and after $20 \mathrm{~min}$ incubation at $37{ }^{\circ} \mathrm{C}$ and $5 \% \mathrm{CO}_{2}$, the TEER value was measured with an EVOM2 resistance meter (WPI). Before the measurement, the STX2 electrode was immersed in a $75 \%$ ethanol solution for $15 \mathrm{~min}$, and then washed with Hank's solution and equilibrated for $5 \mathrm{~min}$. The electrodes were sequentially inserted into the well of a 24 well transwell culture plates to detect the resistance value, and each of the three reserved positions of each well was measured. The calculation formula is as follows:

$$
\mathrm{TEER}=\left(R-R_{0}\right) \times S
$$

$S:$ the effective membrane area $\left(\mathrm{cm}^{2}\right), R$ : the measured resistance value $(\Omega), R_{0}$ : the blank resistance value of the insert without cells $(\Omega)$

\subsection{Permeability test of transmembrane transport marker}

Fluorescein isothiocyanate (FITC)-dextran (FD-4, SigmaAldrich) (1 mg mL ${ }^{-1}$ ) or $200 \mu \mathrm{M}$ propranolol (Sigma-Aldrich) were dissolved in Hank's solution. The above permeability indicator solution $(0.1 \mathrm{~mL})$ was added to the AP side with $0.6 \mathrm{~mL}$ Hank's solution in the BL side. After 2 hours incubation, the fluorescence intensity of FD-4 was measured by a microplate reader (Molecular Devices Incorporated, Sunnyvale, USA) at 480/ $520 \mathrm{~nm} .{ }^{30}$ Propranolol content was determined by high performance liquid chromatography (HPLC), the column was a Macherey-Nagel C-18 reversed-phase column $(250 \mathrm{~mm} \times 4.6$ $\mathrm{mm}, 5 \mu \mathrm{m})$. The chromatographic conditions were as follows: column temperature $37^{\circ} \mathrm{C}$, mobile phase was phosphate solution ( $25 \mathrm{mM}, \mathrm{pH} 6.00)$ : acetonitrile (70:30), the flow rate is 1 $\mathrm{mL} \min ^{-1}$, the injection volume is $10 \mu \mathrm{L}$, and the detection 
wavelength is $225 \mathrm{~nm}$. Apparent permeability coefficient $\left(P_{\text {app }}\right)$ was calculated according to the following equation:

$$
P_{\text {app }}=\Delta Q \times V /\left(\Delta t A C_{0}\right)
$$

$\Delta Q$ : concentration of the BL side in $\Delta t$, the unit is $\mathrm{mg} \mathrm{mL}^{-1}$ (or $\mu \mathrm{M}), V$ : volume of the solution on the BL side, and the unit is $\mathrm{mL}$ (or $\mathrm{L}$ ), $A$ : effective membrane area of the insert, $C_{0}$ : initial concentration of the compound in $\mathrm{mg} \mathrm{mL}^{-1}$ (or $\mu \mathrm{M}$ ).

\subsection{Determination of alkaline phosphatase (ALP) activity}

The test was performed according to the ALP kit instruction and samples were taken from both sides of the transwell membrane on days of 3, 7, 11, 16 and 21 after Caco-2 cell inoculation. The absorbance at $520 \mathrm{~nm}$ was measured by a microplate reader (Molecular Devices), and the ALP activity at different time points was calculated as follows:

$$
\begin{aligned}
& \text { ALP activity in culture medium (King's unit/100 mL) } \\
& \begin{aligned}
= & \frac{A_{\mathrm{t}}-A_{\mathrm{c}}}{A_{\mathrm{s}}-A_{\mathrm{c}}} \times \text { phenol standard concentration }\left(0.02 \mathrm{mg} \mathrm{mL}^{-1}\right) \\
& \quad \times 100 \mathrm{~mL} \times \text { sample dilution multiple }
\end{aligned}
\end{aligned}
$$

where King's unit is defined as product of $1 \mathrm{mg}$ of phenol in $100 \mathrm{~mL}$ of liquid reacted at $37^{\circ} \mathrm{C}$ for $15 \mathrm{~min} ; A_{\mathrm{t}}$ is the measured OD value; $A_{\mathrm{s}}$ is the standard OD value, and $A_{\mathrm{c}}$ is the OD value of the blank.

\subsection{Determination of proliferation inhibition rate and survival rate of Caco-2 cells}

Cell proliferation inhibition rate and survival rate were determined by CCK- 8 method..$^{31}$ The cell density was adjusted to $4 \times$ $10^{4}$ cells per $\mathrm{mL}$, and $200 \mu \mathrm{L}$ per well was seeded in a 96 well plate. When the cells were in the logarithmic phase after 24 hours of adherent growth, the medium was discarded, and 200 $\mu \mathrm{L}$ of the medium containing DON/kaempferol was added to continue the culture for another 24 hours. One hour before the end of the culture, the medium was also discarded, and $10 \%$ CCK-8 solution (Beyotime Biotechnology, Shanghai, China) was added to $100 \mu \mathrm{L}$. After incubation at $37^{\circ} \mathrm{C}$ for $1 \mathrm{~h}$, the absorbance A was measured at $450 \mathrm{~nm}$. Cell proliferation inhibition rate $Y=\left[1-\left(A_{\mathrm{y}}-A_{\mathrm{bx}}\right) /\left(A_{\mathrm{c}}-A_{\mathrm{bz}}\right)\right] \times 100 \%$, wherein bx and bz are the corresponding blank group, $\mathrm{c}$ is the negative control group, and $\mathrm{y}$ is the drug treatment group.

Kaempferol pretreatment refers to the cells pre-incubated with kaempferol for $24 \mathrm{~h}$, followed by treatment with DON for another $24 \mathrm{~h}$. Method for determining the survival rate is the same as the inhibition rate of proliferation. The calculation formula is cell survival rate $=100 \%$ - cell proliferation inhibition rate.

\subsection{Western blot}

The culture medium was removed, and the cells were washed twice with $4{ }^{\circ} \mathrm{C}$ pre-cooled PBS, then $80 \mu \mathrm{L}$ of RIPA potent lysate (Beyotime) containing $1 \mathrm{mM}$ protease inhibitor PMSF (Beyotime) was added to each insert of the 24 well transwell plate. After lysed for $2 \mathrm{~min}$, the cells were scraped down into $1.5 \mathrm{~mL}$ pre-cooled centrifuge tubes. Centrifuged at $14000 \times g$ for $5 \mathrm{~min}$ at $4{ }^{\circ} \mathrm{C}$, the supernatant was stored at $-80^{\circ} \mathrm{C}$ for testing. All of the above operations were performed on ice. The protein concentration was determined using a BCA protein concentration assay kit (Beyotime).

Prepare the sample solution according to the instructions of the NuPAGE® MOPS SDS Buffer Kit. After mixing, the protein in solution was denaturated at $70^{\circ} \mathrm{C}$ for $10 \mathrm{~min}$. The preformed gel was placed in an electrophoresis tank, and then $200 \mathrm{~mL}$ of $5 \%$ NuPAGE® MOPS SDS loading buffer was added to each of the front and rear tanks, and $500 \mu \mathrm{L}$ of NuPAGE $®$ antioxidant was added to the front tank. Pull out the comb and add $20 \mu \mathrm{L}$ of each sample. Electrophoresis lasts about 75 minutes at a voltage of $200 \mathrm{~V}$ and an electric current of $80 \mathrm{~mA}$. When the electrophoresis was completed, the gel was taken out and transferred at $20 \mathrm{~V}, 1 \mathrm{~min} ; 23 \mathrm{~V}, 4 \mathrm{~min} ; 25 \mathrm{~V}, 2 \mathrm{~min}$, and then blocked with a TBST containing $5 \%$ BSA at room temperature for $2 \mathrm{~h}$ under shaking.

After incubating with a suitable concentration of primary antibody for claudin-3, claudin-4, occludin and ZO- 1 at $4{ }^{\circ} \mathrm{C}$ overnight, the primary antibody dilution was discarded and washed 6 times with TBST on a shaker for 10-20 min each time. The secondary antibody dilution was added and incubated for $2 \mathrm{~h}$ at room temperature, then the secondary antibody dilution was removed and washed again with TBST on a shaker for 6 times. After color developed, exposed and photographed, grayscale analysis was performed using Image J software (Bethesda softworks LLT, Bethesda, USA).

\subsection{Statistical analysis}

Significant analysis of differences between groups were performed using SPSS software (IBM Corporation, Armonk, USA), and $p<0.05$ was set as the level of statistical difference. Charts in the article were plotted using Origin 8.5 (OriginLab Corp., MA, USA). The data were presented as mean \pm standard deviation. The heatmap analysis was drew with $\mathrm{R}$ package of ggplot2.

\section{Results}

\subsection{Characteristics of Caco- 2 cell model in different stages}

Caco-2 cell line was from human colorectal cancer cells, and cells grown on a porous permeable membrane after inoculation at a specific density can fuse and differentiate to form a continuous monolayer. Because of its similarity to differentiated intestinal epithelial cells (microvilli structure, enzymes associated with the intestinal brush border epithelium, cell polarity and tight junctions), it is commonly used to simulate the human intestinal epithelium after a Caco-2 monolayer was established.

3.1.1. Integrity and permeability. The transepithelial electric resistance (TEER) and the flux rate of fluorescent FD-4 are two complementary markers to characterizes the permeability of the Caco- 2 cell monolayer. It can be seen from Fig. 1 that the Caco-2 cells have a low resistance value and a high FD-4 flux rate in the beginning of culture until a stable value of TEER $(250 \Omega$ 

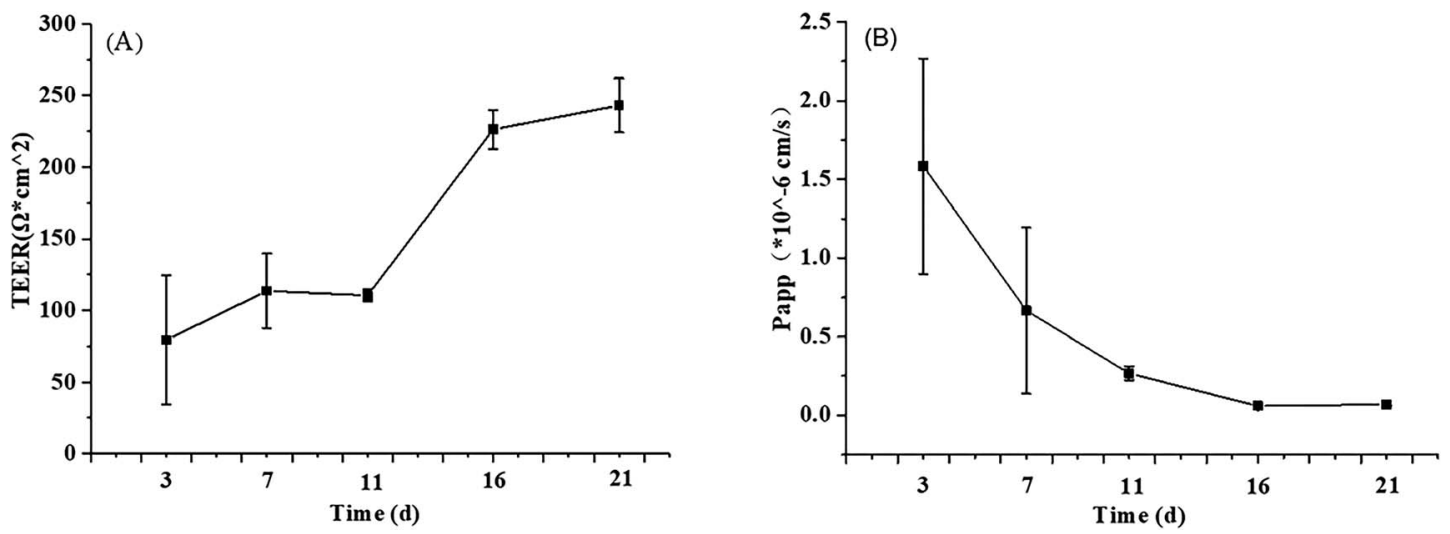

Fig. 1 The TEER value (A) and FD-4 permeability (B) at different stages of Caco-2 cell proliferation and differentiation. The data were presented as mean \pm SD of at least three replicates.

$\left.\mathrm{cm}^{2}\right)$ and FD-4 flux rate $\left(P_{\text {app }}: 10^{-7} \mathrm{~cm} \mathrm{~s}^{-1}\right)$ that are characterized of Caco-2 cell monolayer achieved after culture for 21 days. Clearly, there were two different stages according to the TEER values: the first stage from 3-11 days representing the proliferative stage with lower TEER, and the second stage from 11-21 days representing the proliferative/differentiation stage. Apparently, the 11th day was likely a turning point from cell proliferation to differentiation, and so the cell cultures at days of 3rd, 11th and 21st were chosen as the time points to investigate the changes of tight junction proteins under different conditions. The experimental result was also consistent with the general belief that the first 7-10 days after Caco- 2 cell inoculation is the rapid proliferation and differentiation stage of the cells, and the last 10 days is the functional perfect stage of the cells including cell polarization and the construction of the active transport systems in differentiated cells.

Another risk in the process of Caco-2 cell modeling is the possibility of forming multilayer cells rather than the monolayer model required by the research. Propranolol, as a marker of intracellular transport, was used to verify the integrity of Caco-2 cell monolayer according to its $P_{\text {app }}$ value $(>10 \times$ $\left.10^{-6} \mathrm{~cm} \mathrm{~s}^{-1}\right)$. In the current study, the average $P_{\text {app }}$ value of propranolol determined by HPLC was $13.69 \times 10^{-6} \mathrm{~cm} \mathrm{~s}^{-1}$ (ESI Fig. $\mathrm{A}$ and $\mathrm{B} \dagger$ ), which is greater than the standard, demonstrating that the permeability of the Caco- 2 cells model meets the experimental standard. ${ }^{32}$

3.1.2. Activity of alkaline phosphatase (ALP) in Caco-2 cells at different stages of proliferation and differentiation. During the differentiation process of Caco-2 cells, their polarization will form apical side (AP) of villous surface and basolateral side (BL) of basal surface. Alkaline phosphatase (ALP) is a marker of brush border formation in AP side of small intestinal epithelium. When ALP was used as an indicator of cell differentiation, its activity increased gradually along the time on AP side, but no changes of ALP on the BL side was observed (Fig. 2), indicating the polarity of Caco-2 cells was successfully established at the end of 21st day. In the meantime, different properties of ALP (start point, rapid increase phase, and stable stage) were shown from its activity profile along the differentiation process, and its activity at the time points of 3rd, 11th, and 21st day, just as reflected from TEER, can be considered as another indicator of Caco-2 cell proliferation and differentiation.

\subsection{Determination of DON and kaempferol (KAM) treatment concentration}

The differentiation of Caco- 2 cells is a continuous process involving cell proliferation and differentiation. Thus, the Caco-2 cell differentiation affected by DON and KAM was studied according the selected time scheme to determine the dosage for later TJ protein expression studies. When the cell viability (proliferation stage) was measured after incubation in a complete culture medium containing different concentrations of DON or kaempferol for 24 hours, different profiles were shown for DON and KAM. The inhibition rate of DON was tightly dependent on the concentration of DON (Fig. 3A), and polynomial fit analysis demonstrated that the half-maximal inhibitory concentration $\left(\mathrm{IC}_{50}\right)$ of DON for Caco-2 cell

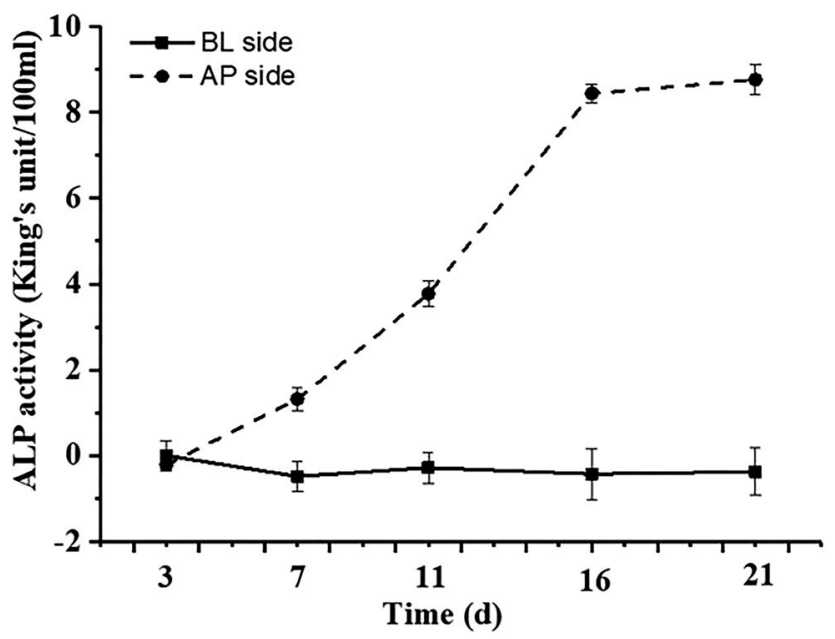

Fig. 2 Alkaline phosphatase activity at different stages of proliferation and differentiation of Caco- 2 cells modeling. AP: apical side, BL: basolateral side. The data were presented as mean \pm SD of at least three replicates. 

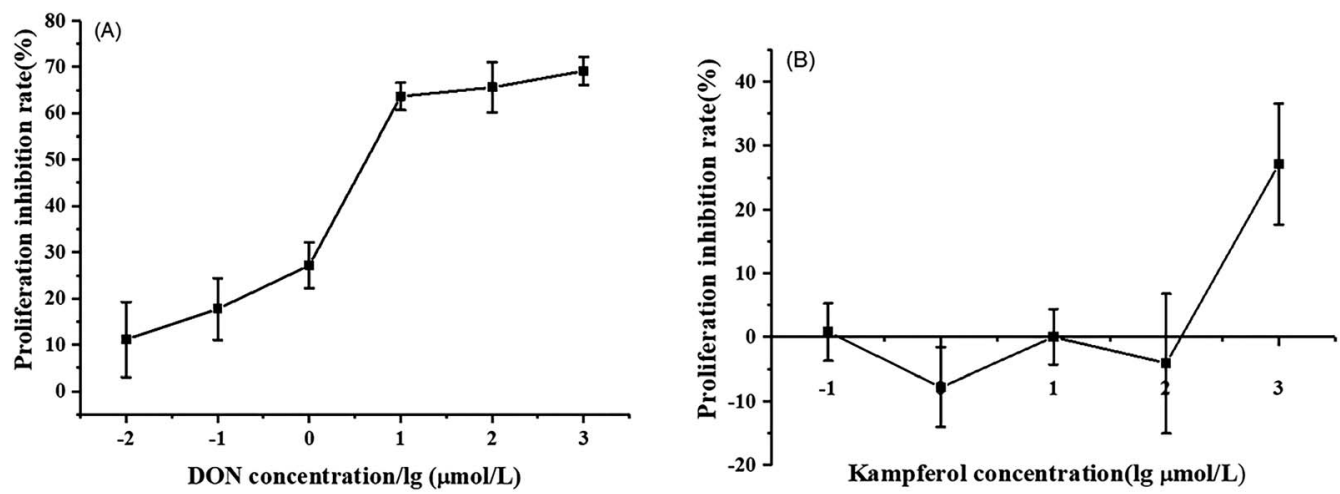

Fig. 3 Effects of DON (A) and kaempferol (B) on proliferation inhibition rate of Caco-2 cells. The data were presented as mean \pm SD of at least three replicates.

proliferation was $8.50 \mu \mathrm{M}$. For kaempferol, the minimal concentration to inhibit Caco-2 cell growth was $100 \mu \mathrm{M}$ (Fig. 3B). Thus, DON is more toxic than KAM concerning the cell viability, and the negative inhibition rate at lower concentrations of $\operatorname{KAM}(<100 \mu \mathrm{M})$ suggests it may even be beneficial to the cell growth.

For differentiated Caco-2 cells, the parameter of cell permeability was applied to evaluate the impact of DON and
KAM on the integrity of cell-to-cell adhesion or tight junction. Obviously, the DON treatment substantially reduced the transepithelial electrical resistance (TEER) values in a concentrationdependent manner $(\geq 5 \mu \mathrm{M})$, which is consistent with the conversely increased FD-4 flux rate (Fig. 4A and B). However, in the KAM treatment (up to $200 \mu \mathrm{M}$ ) groups, no significant impact on cell permeability was observed, implying that KAM would
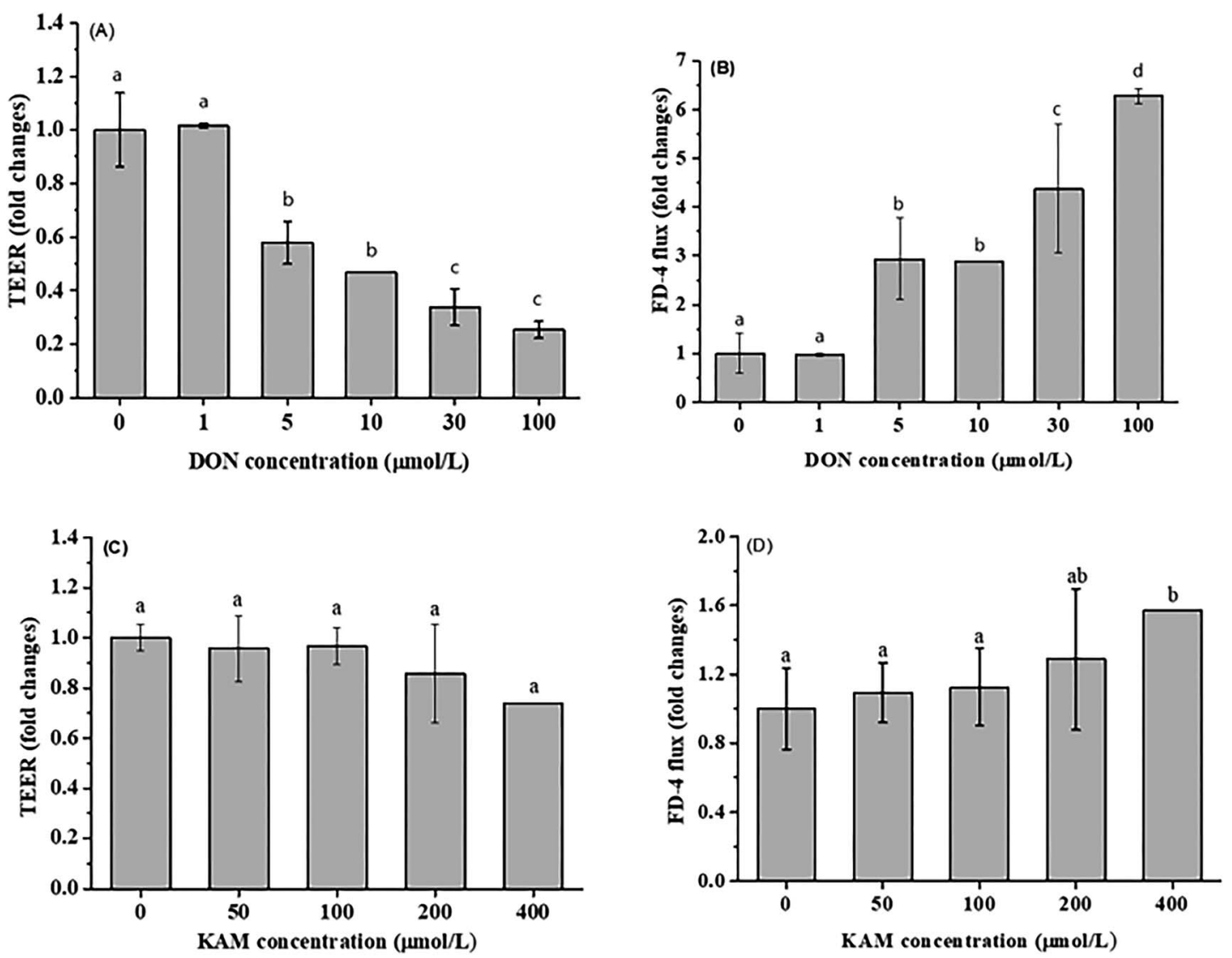

Fig. 4 Effect of DON and KAM on the permeability (normalized by control) of Caco-2 cell monolayer after treatment by DON (A and B) or KAM (C and $\mathrm{D}$ ) for $24 \mathrm{~h}$. Fold changes referred to the ratio with initial values measured before treatment. The data were presented as mean \pm SD of at least three replicates. Different letters represent a significant difference at a level of $p<0.05$. 
not adversely affect the Caco- 2 cell monolayer in the used dosage (Fig. 4C and D).

In summary, by considering the above effects of DON and KAM on the proliferation and the permeability of differentiated monolayer Caco-2 cells as well as the existed literature reports, ${ }^{29,33}$ a dosage of $5 \mu \mathrm{M}$ DON and $100 \mu \mathrm{M}$ KAM were used to study the effects of DON and KAM on the tight junction of Caco-2 cells at different stages of proliferation and differentiation.

\subsection{Tight junction protein expression in Caco-2 cells at different stages of proliferation and differentiation}

The permeability of monolayer Caco- 2 cell is intimately associated to the cell adhesion, particularly the tight junction which is a network formed by many different proteins. In order to gain a complete understanding, occludin and claudins (3 and 4) representing the transmembrane protein, and peripheral adaptor protein of zonula occludens-1 (ZO-1) were selected as the targets to investigate the impact of DON and KAM on the integrity of $\mathrm{TJ}$ at different stages (proliferative stage at 3rd day, turning point at 11th day, and differentiated stage at 21st day).

3.3.1. Effects of DON and kaempferol on the tight junction protein expression in proliferative Caco-2 cells. On the 3 rd day after the cells were inoculated into the insert, culture medium containing DON, KAM and KAM + DON (first $100 \mu \mathrm{M}$ KAM 24 hours, then $5 \mu \mathrm{M}$ DON 24 hours) were added to both sides of the insert to treat the cells. It can be seen from Fig. 5A and B that the TEER of the blank control rapidly increased to 5.76 times of the initial value (2 days before treatment), and the flux of FD-4 was also reduced to about $10 \%$ of the initial value. However, both DON and KAM significantly reduced the TEER. Particularly, the addition of DON after KAM pretreatment lead to a dramatic reduction of TEER resulted from a high permeability of the cells, and KAM pretreatment worsened the permeability compared to DON alone.

When the TJ protein expression was examined (Fig. 5C and D), KAM treatment alone significantly reduced the protein expression of occludin, and DON significantly reduced the expression of claudin- 4 and occludin, which is consistent with the decreased TEER and high flux rate of FD-4. KAM pretreatment did not improve the expression of claudin-4 adversely affected by DON, but the protein expression of claudin-3 was increased significantly. When the correlation between TEER and protein expression was analyzed, the expression level of claudin-4 is significantly correlated with the cell permeability, indicating the TJ protein of claudin-4 is associated with the cell permeability at the proliferation stage. DON treatment not only reduced claudin-4, but also reduce the cell proliferation (Fig. 3), and both can cause a high permeability. Although KAM is better than DON (probably due to no inhibition for cell proliferation), KAM pretreatment worsened the TEER (maybe related to ZO-1)
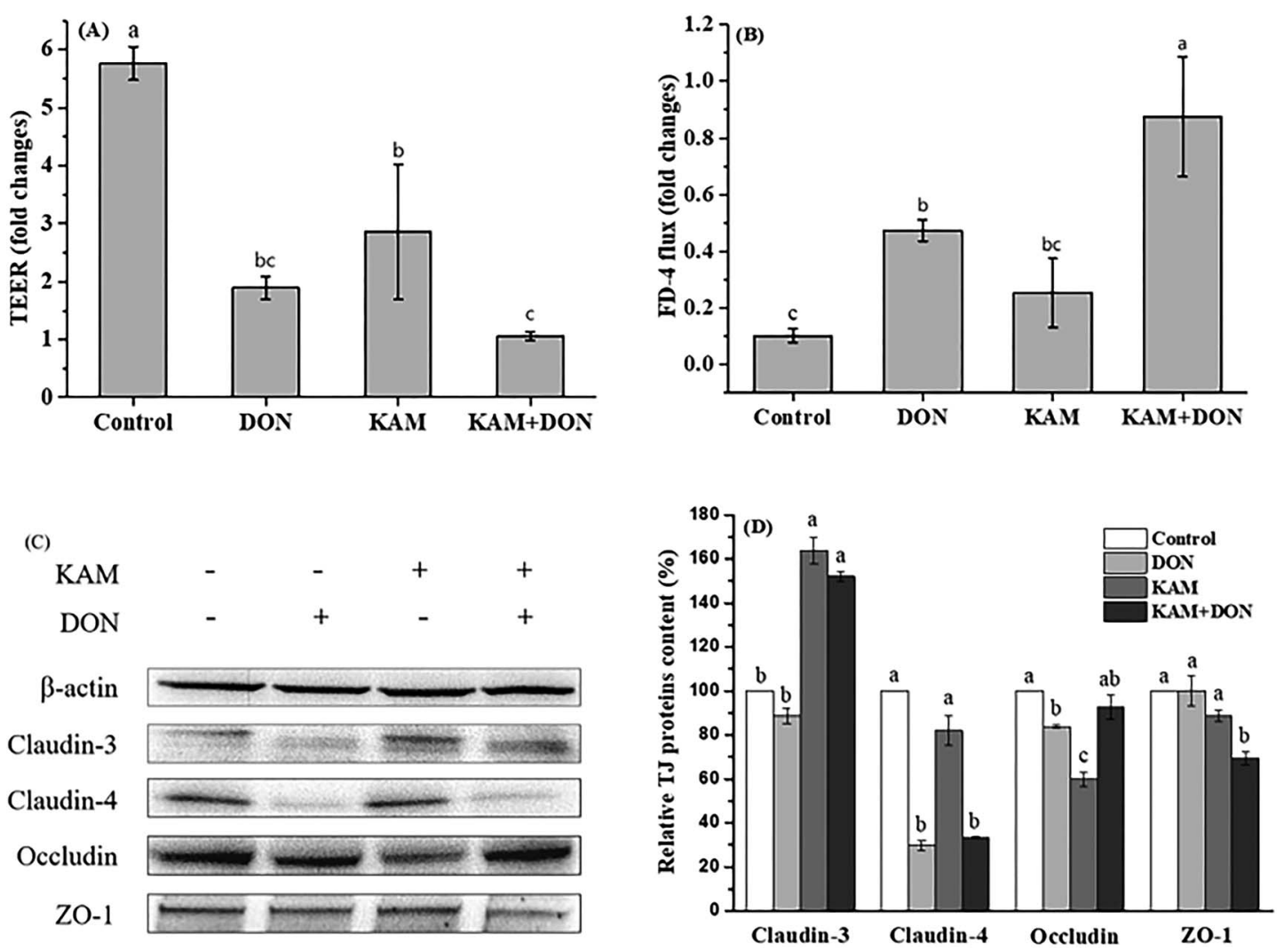

Fig. 5 Effects of KAM pretreatment on the permeability ( $A$ and $B$ ) and tight junction protein expression ( $C$ and $D)$ in Caco-2 cells during the proliferation stage ( 3 days after inoculation). The fold changes refer to the ratios relative to initial values before treatment. The data were presented as mean \pm SD of at least three replicates. Different letters of the same parameter indicating significant differences at the 0.05 level. 
with an increase of claudin-3, indicating claudin-3 is not related to the permeability at the proliferation stage of the Caco- 2 cells. Thus, both inhibiting cell proliferation and impairing tight junctions are likely the main reasons for the adverse effect of DON and KAM on the cell permeability in the early stage of Caco-2 cell differentiation.

3.3.2. Effects of DON and KAM on the tight junction protein expression in proliferative/differentiated Caco-2 cells. When the 11th day Caco-2 cells were treated by DON and KAM as well as KAM + DON, the TEER value of the DON and KAM + DON group significantly increased compared to the control and KAM group, especially the KAM pretreatment group with the highest TEER and lowest FD-4 flux rate (Fig. 6A and B). However, the TEER and FD-4 flux rate were not as before showing an inverse relationship, particularly for KAM group. These results were completely reversed when compared to the Caco- 2 cells in their early proliferative stage.

The TJ protein expression pattern after treatments also showed a substantial difference from the proliferative Caco-2 cells (Fig. 6C and D). In DON alone treatment group, except a slight reduced occludin expression, the expression of claudin4 was significantly increased. For KAM group, the occludin expression was significantly increased, and a higher level of claudin-3 was also observed (compared to the control). After kaempferol pretreatment, no changes were found for the expression levels of claudin-4, occludin and ZO-1, but the expression of claudin-3 was significantly increased, which was consistent with the permeability of KAM pretreatment group. Altogether, no reduction of TJ protein expression was found in the treatment group compared to the control, which is substantially different from the proliferative Caco- 2 cells. When the relationship between TEER and TJ protein was analyzed, it seems both claudin-4 (high TEER in DON alone) and claudin-3 (high TEER in KAM pretreatment group) contribute to the reduced permeability of Caco- 2 cells in the proliferative/ differentiated stage.

The disappearance of inverse relationship between TEER and FD-4 flux rate and positive effect of both DON and KAM on TJ protein expression signify the uniqueness of the Caco- 2 cell model at the 11th day, which might represent the separation point of cell proliferation and differentiation. Although we do not have a good explanation for the observed phenomenon, a higher tolerance to environments or the basic biological mechanism of compensating changes for adaption and survive might played a role in this special stage of Caco- 2 cell differentiation.

3.3.3. Effects of DON and KAM on the tight junction protein expression in differentiated Caco-2 cell monolayer. After the proliferation and differentiation for 21 days, the Caco2 cells become a monolayer that is similar to the epithelium in the small intestine. As shown in Fig. 7A and B, DON significantly decreased the TEER value with increased FD-4 flux while
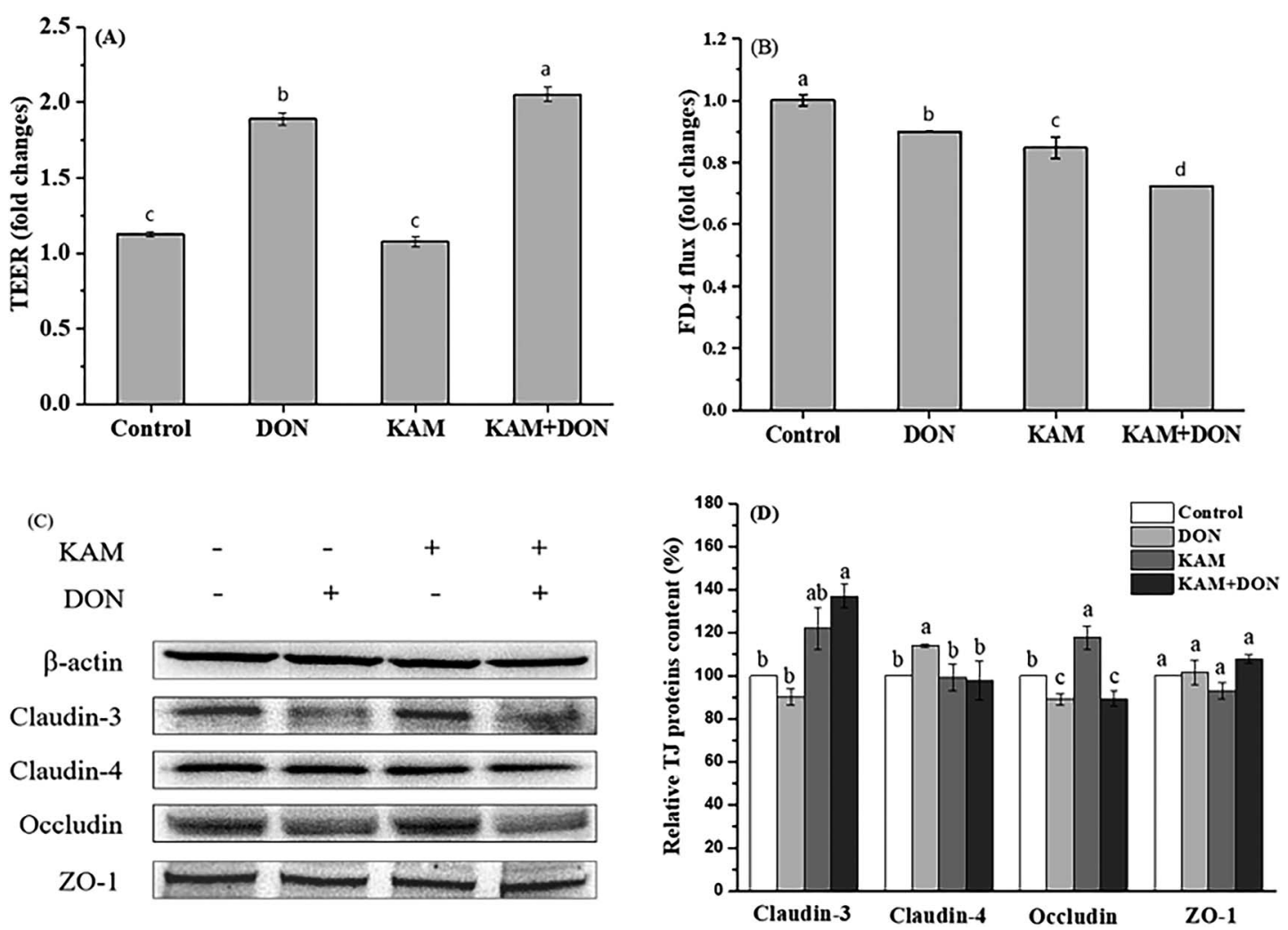

Fig. 6 Effects of KAM pretreatment on the permeability ( $A$ and B) and tight junction protein expression (C and D) in Caco-2 cells during the intermediate stage of differentiation (11 days after inoculation). The fold changes refer to the ratios relative to initial values before treatment. The data were presented as mean \pm SD of at least three replicates. Different letters of the same parameter indicating significant differences at the 0.05 level. 

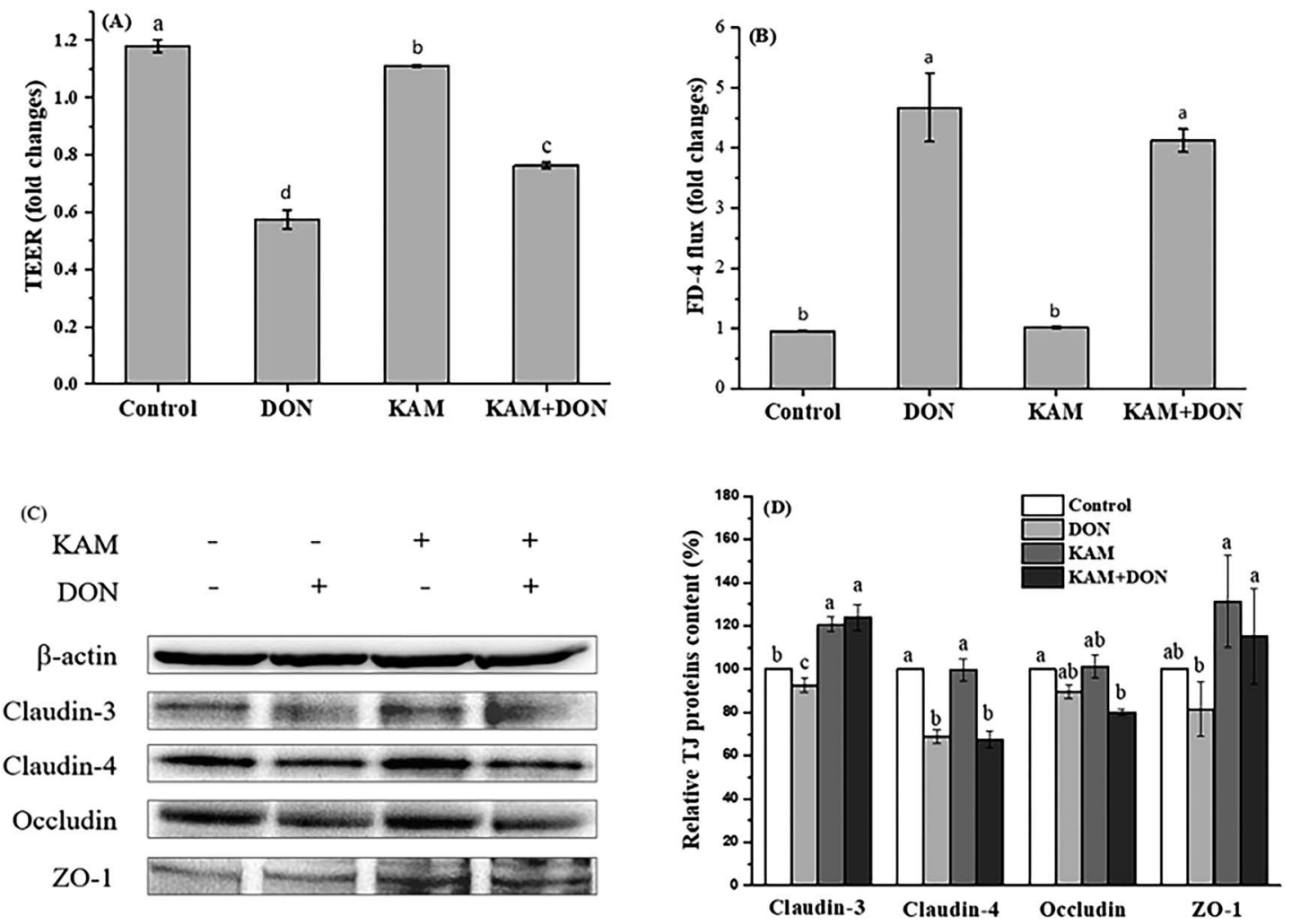

Fig. 7 Effects of KAM pretreatment on the permeability (A and B) and tight junction protein expression (C and D) in differentiated Caco-2 cell monolayer (21 days after inoculation). The fold changes refer to the ratios relative to initial values before treatment. The data were presented as mean \pm SD of at least three replicates. Different letters of the same parameter indicating significant differences at the 0.05 level.

the monolayer permeability in KAM group is comparable to the control, and KAM pretreatment also significantly improved the TEER and reduced the adverse effect on monolayer permeability caused by DON. Apparently, although the same treatment conditions were applied to Caco-2 cells, the resulted monolayer permeability is different from either the cells at proliferative stage or at the intermediates stage of differentiation.

From the results of protein expression (Fig. 7C and D), DON alone caused a noteworthy decrease in claudin- 4 , while KAM alone and its pretreatment significantly increased the protein expression of claudin-3 and ZO-1 without affecting the expression of claudin-4.

3.3.4. Correlation between permeability and TJ protein expression. The permeability of $\mathrm{Caco}-2$ monolayer under different stages of differentiation after treatment of mycotoxin of DON and phenolic compound of KAM was investigated. The correlation analysis between the permeability and TJ protein revealed that claudin- 4 is the core $\mathrm{TJ}$ protein to the permeability of the Caco-2 cell monolayer, and it is also the target protein of DON's cytotoxicity affecting the TJ integrity of Caco-2 cells at different stages. For KAM, although it specifically promoted the expression of claudin-3, the permeability of the Caco-2 cell monolayer did not significantly correlated with the expression of claudin-3. The cluster analysis (Fig. 8) showed that TEER of Caco2 cell monolayer (differentiated) is more closely associated with claudin-4 and occludin and FD-4 is more closely related to ZO-1 and claudin-3. In the Caco-2 cell at 11th days, TEER is more closely with claudin-4 and ZO-1, and FD-4 is more closely with claudin-3 and occludin. For the proliferative Caco-2 cells, a completely different cluster relationship was observed except the TEER and claudin-4. From the viewpoint of treatments, KAM and control is likely one cluster while DON and KAMpretreatment is another cluster. Thus, the impact of DON and KAM on the permeability of the Caco-2 cells is strongly dependent on its differentiation stages and the expression of claudin- 4 .

\section{Discussion}

The intestinal epithelium, which is composed of a monolayer of cells, acts not only as the primary site for nutrient uptake but also as the barrier to the external environment. The rapid turnover of the monolayer cells needs a continuous dividing of intestinal stem cells resided at the bottom of crypts. The divided stem cells then enter the transit-amplifying zone where they proliferate and differentiate until they migrate to the villus tip as matured cells. Certainly, the microenvironment formed by luminal materials, particularly food materials in the lumen, will have a great impact on cell proliferation and differentiation. The phenolic compounds of KAM rich in fruit and vegetable is a common food component with a variety of health benefits. Mycotoxin of DON, which is toxic to human health, is becoming a food hazard problem due to its wide contamination in many 

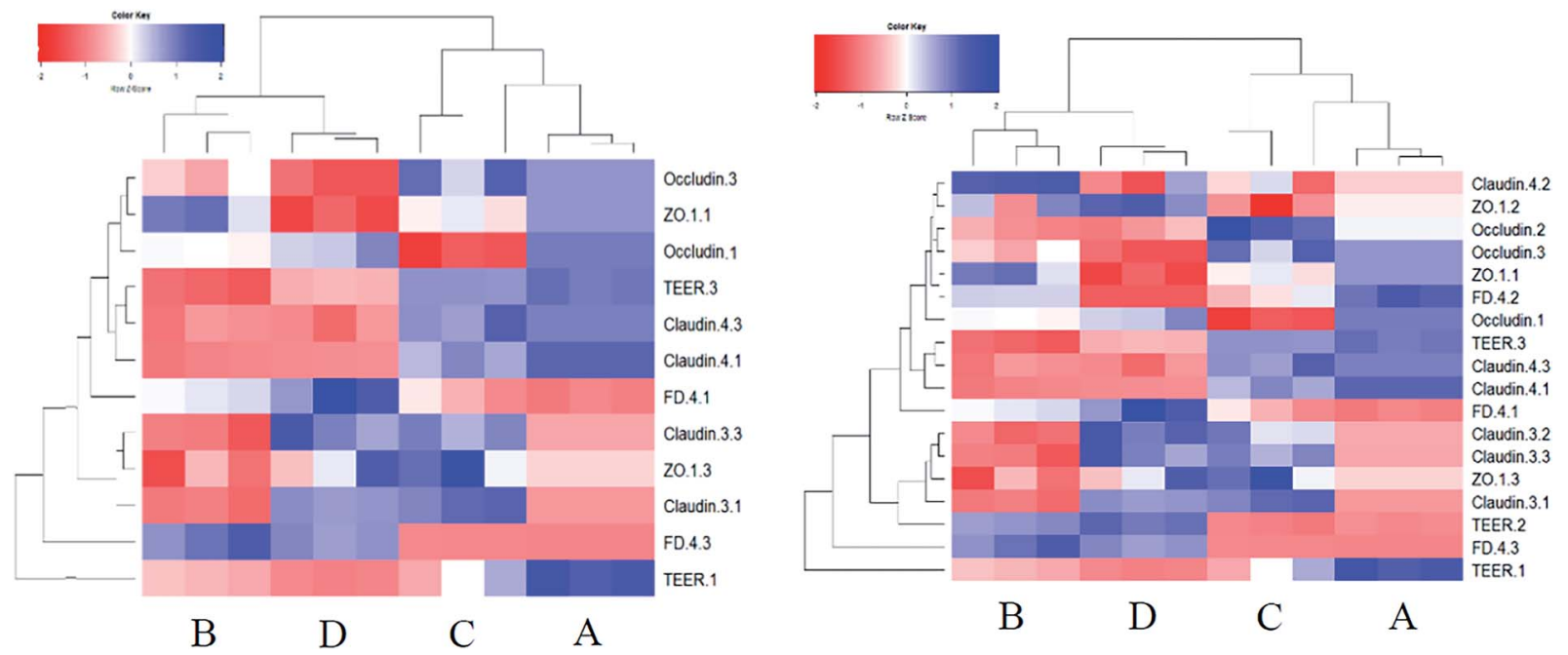

Fig. 8 The heatmap analysis of the TJ protein expression and the permeability of the Caco-2 cell models at stages of proliferation and differentiated state (left side), and including the intermediate stage (11th day). A: control, B: DON, C: KAM, D: KAM + DON. The number after the parameter represents different stages (1: 3rd day, 2: 11th day, 3: 21st day).

crops and food products. Thus, the antagonistic effect of KAM on the cytotoxicity DON affecting the epithelium barrier function, as the first step to enter into the body, was investigated by mimicking the process of intestinal cell proliferation and differentiation as they moved from the base of the crypt to the villus tip.

The toxic effect of DON on the Caco-2 cell monolayer was evidenced by a dose-dependent decrease of the TEER values at a concentration of $\geq 5 \mu \mathrm{M}$ (Fig. 4). Further study demonstrated the TJ protein expression of claudin- 4 and claudin- 3 were significantly reduced in differentiated monolayer stage (Fig. 7). This result is consistent with literature reports using Caco-2 cell monolayer model $^{5}$ and porcine intestinal epithelial cell line (IPEC-1). ${ }^{13}$ Regarding the mechanism, the inhibition of protein synthesis and activation of a mitogen-activated protein kinases (MAPK) signaling pathway, specifically the reduced TJ protein claudin-4 all contribute to the toxic effect of DON to the epithelium barrier function., ${ }^{5,13,34}$ However, we also observed that the impact of DON one Caco-2 cell permeability and the expression on claudin-4 is dependent on the stages of Caco-2 cell proliferation and differentiation. DON inhibited the expression of claudin-4 at its proliferation and differentiated (monolayer) stages, but increased the claudin- 4 expression at 11th day, which is an intermediate stage of Caco-2 cell differentiation. Although we do not know the exact reason, it does strengthen the relationship between permeability and the expression of claudin- 4 .

In contrast to the development stage-dependent toxicity of DON, KAM did not affect the expression of claudin-4, but always promotes the expression of TJ protein of claudin-3. However, KAM only induced a leveled permeability to the control, which is not correlated to the expression of claudin-3 (Fig. 5-7). However, for the differentiated Caco-2 cell monolayer, KAM pretreatment did significantly reduced the permeability after DON exposure (Fig. 7 KAM + DON) without influencing the level of claudin-4. Actually, there have been reports on the beneficial effects on epithelium barrier of many polyphenol compounds including berberine, grape seeds extract, quercetin, green tea polyphenols through MAPK activation and inhibiting inflammation induced by NF- $\mathrm{\kappa B}$ as well as scavenging ROS. ${ }^{35}$ Similarly, KAM also regulates MAPKs and NF- $\mathrm{KB}$ signaling pathways to induce many health benefits, ${ }^{36}$ and it should be beneficial to the barrier function of epithelium. Indeed, there have been numerous studies indicating that claudin- 3 is a barrier-forming TJ protein. ${ }^{37}$ The permeability of Caco-2 cells grown on transwell filters was reduced by mycotoxin of ochratoxin A treatment, which is due to reduced expression of claudin-3 and $4 .^{38} \mathrm{An}$ increase of gastric epithelium permeability by oxidative stress is also associated with a reduction of claudin-3 protein. ${ }^{39}$ Thus, pretreatment by KAM, which specifically upregulates the expression of claudin-3, may have the potential to reduce the damage to epithelium permeability caused by DON. In the meantime, KAM treatment also upregulate the expression of ZO-1 that may also contribute the reduced permeability of Caco2 cell monolayers.

\section{Conclusion}

In this study, the response of tight junctions of Caco-2 cells at different stages of proliferation and differentiation to mycotoxin DON and phenolic compound kaempferol was studied. The results showed that DON and kaempferol affect the cell proliferation in a dose-dependent manner, and the permeability of the Caco-2 TJ affected by DON and KAM is highly dependent on the stage of cell proliferation and differentiation. Further research found that claudin-4 is the target protein of DON while claudin-3 is specifically upregulated by KAM, and KAM pretreatment significantly increase the integrity of differentiated Caco-2 cell monolayer. Thus, KAM, through upregulating 
claudin-3 and ZO-1, may have a high potential to improve the epithelium integrity damaged by DON exposure.

\section{Conflicts of interest}

There are no conflicts to declare.

\section{Acknowledgements}

The research was funded by National Natural Science Foundation of China No: 31471585.

\section{References}

1 E. F. S. Authority, EFSA J., 2013, 11, DOI: 10.2903/ j.efsa.2013.3379.

2 J. J. Pestka and A. T. Smolinski, J. Toxicol. Environ. Health, Part B, 2005, 8, 39-69.

3 B. Grenier and T. J. Applegate, Toxins, 2013, 5, 396-430.

4 J. J. Pestka, Arch. Toxicol., 2010, 84, 663-679.

5 J. V. De Walle, T. Sergent, N. Piront, O. Toussaint, Y. J. Schneider and Y. Larondelle, Toxicol. Appl. Pharmacol., 2010, 245, 291-298.

6 P. Pinton, J. P. Nougayrede, J. C. Del Rio, C. Moreno, D. E. Marin, L. Ferrier, A. P. Bracarense, M. Kolf-Clauw and I. P. Oswald, Toxicol. Appl. Pharmacol., 2009, 237, 41-48.

7 P. Pinton, D. Tsybulskyy, J. Lucioli, J. Laffitte, P. Callu, F. Lyazhri, F. Grosjean, A. P. Bracarense, M. Kolf-Clauw and I. P. Oswald, Toxicol. Sci., 2012, 130, 180-190.

8 A. Springler, S. Hessenberger, G. Schatzmayr and E. Mayer, Toxins, 2016, 8, DOI: 10.3390/toxins8090264.

9 S. Tsukita, H. Tanaka and A. Tamura, Trends Biochem. Sci., 2019, 44, 141-152.

10 P. Artursson and S. Knight, Science, 2015, 716-717.

11 M. S. Balda and K. Matter, Curr. Opin. Cell Biol., 2016, 42, 94101.

12 P. Rowart, J. Wu and M. J. Caplan, Int. J. Mol. Sci., 2018, 19, DOI: $10.3390 /$ ijms19072040.

13 P. Pinton, C. Braicu, J. P. Nougayrede, J. Laffitte, I. Taranu and I. P. Oswald, J. Nutr., 2010, 140, 1956-1962.

14 T. Suzuki and H. Hara, J. Nutr., 2009, 139, 965-974.

15 T. Suzuki and H. Hara, J. Nutr. Biochem., 2011, 22, 401-408.

16 M. C. Valenzano, K. DiGuilio, J. Mercado, M. Teter, J. To, B. Ferraro, B. Mixson, I. Manley, V. Baker, B. A. Moore, J. Wertheimer and J. M. Mullin, PLoS One, 2015, 10, e0133926.

17 B. Lee, K. M. Moon and C. Y. Kim, J. Immunol. Res., 2018, 2018, 2645465.
18 M. B. G. Kiewiet, M. I. Gonzalez Rodriguez, R. Dekkers, M. Gros, L. H. Ulfman, A. Groeneveld, P. de Vos and M. M. Faas, Food Funct., 2018, 9, 4164-4172.

19 P. Akbari, S. Braber, A. Alizadeh, K. A. Verheijden, M. H. Schoterman, A. D. Kraneveld, J. Garssen and J. FinkGremmels, J. Nutr., 2015, 145, 1604-1613.

20 S. Noda, S. Tanabe and T. Suzuki, J. Funct. Foods, 2014, 10, 112-116.

21 S. Noda, S. Tanabe and T. Suzuki, Mol. Nutr. Food Res., 2013, 57, 2019-2028.

22 L. Gu, N. Li, Q. Li, Q. Zhang, C. Wang, W. Zhu and J. Li, Fitoterapia, 2009, 80, 241-248.

23 M. Shigeshiro, S. Tanabe and T. Suzuki, J. Funct. Foods, 2013, 5, 949-955.

24 T. Chen, C. Y. Kim, A. Kaur, L. Lamothe, M. Shaikh, A. Keshavarzian and B. R. Hamaker, Food Funct., 2017, 8, 1166-1173.

25 J. M. Calderón-Montaño, E. Burgos-Morón, C. PérezGuerrero and M. López-Lázaro, Mini-Rev. Med. Chem., 2011, 11, 298-344.

26 A. Sekiguchi, S. I. Motegi, C. Fujiwara, S. Yamazaki, Y. Inoue, A. Uchiyama, R. Akai, T. Iwawaki and O. Ishikawa, J. Dermatol. Sci., 2019, 96, 8-17.

27 S. Cui, J. Tang, S. Wang and L. Li, Biomed. Pharmacother., 2019, 115, 108888.

28 R. M. Hussein, W. R. Mohamed and H. A. Omar, Pestic. Biochem. Physiol., 2018, 152, 29-37.

29 T. Suzuki, S. Tanabe and H. Hara, J. Nutr., 2011, 141, 87-94. 30 N. Li, L. Gu, L. Qu, J. Gong, Q. Li, W. Zhu and J. Li, Eur. J. Pharm. Sci., 2010, 40, 1-8.

31 Y. Sang, W. Li and G. Zhang, Food Funct., 2016, 7, 3703-3715. 32 S. Yee, Pharm. Res., 1997, 763-766.

33 P. Akbari, S. Braber, H. Gremmels, P. J. Koelink, K. A. Verheijden, J. Garssen and J. Fink-Gremmels, FASEB J., 2014, 28, 2414-2429.

34 P. Pinton, J. P. Nougayrède, J. C. Del Rio, C. Moreno, D. E. Marin, L. Ferrier, A. P. Bracarense, M. Kolf-Clauw and I. P. Oswald, Toxicol. Appl. Pharmacol., 2009, 237, 41-48.

35 G. Yang, S. Bibi, M. Du, T. Suzuki and M. J. Zhu, Crit. Rev. Food Sci. Nutr., 2017, 57, 3830-3839.

36 X. Chen, X. Yang, T. Liu, M. Guan, X. Feng, W. Dong, X. Chu, J. Liu, X. Tian, X. Ci, H. Li, J. Wei, Y. Deng, X. Deng, G. Chi and Z. Sun, Int. Immunopharmacol., 2012, 14, 209-216.

37 D. Gunzel and A. S. Yu, Physiol. Rev., 2013, 93, 525-569.

38 J. McLaughlin, P. J. Padfield, J. P. Burt and C. A. O'Neill, Am. J. Physiol. Cell Physiol., 2004, 287, C1412-C1417.

39 K. Hashimoto, T. Oshima, T. Tomita, Y. Kim, T. Matsumoto, T. Joh and H. Miwa, Biochem. Biophys. Res. Commun., 2008, 376, 154-157. 